(2) OPEN ACCESS

\title{
Faecal immunochemical test is superior to symptoms in predicting pathology in patients with suspected colorectal cancer symptoms referred on a $2 \mathrm{WW}$ pathway: a diagnostic accuracy study
}

\author{
Nigel D'Souza (D) , 1,2,3 Theo Georgiou Delisle, 1,3 Michelle Chen, ${ }^{4}$ Sally Benton, ${ }^{5}$ \\ Muti Abulafi (D) ' 1 The NICE FIT Steering Group
}

- Additional material is published online only. To view, please visit the journal online (http://dx.doi.org/10.1136/ gutjnl-2020-321956).

${ }^{1}$ Colorectal Surgery, Croydon University Hospital, Croydon, UK ${ }^{2}$ Colorectal Surgery, Basingstoke and North Hampshire Hospital, Basingstoke, UK

${ }^{3}$ Surgery \& Cancer, Imperial College London, London, UK

${ }^{4}$ Research \& Development, RM Partners, London, UK

${ }^{5}$ Clinical Biochemistry, Royal Surrey County Hospital, Guildford, UK

Correspondence to Mr Muti Abulafi, Colorectal Surgery, Croydon University Hospital, Croydon CR7 7YE, UK: muti.abulafi@nhs.net

Received 24 May 2020 Revised 6 August 2020 Accepted 9 August 2020 Published Online First 21 October 2020

\section{Linked}

- http://dx.doi.org/10.1136/ gutjnl-2020-323118

\section{Check for updates}

(c) Author(s) (or their employer(s)) 2021. Re-use permitted under CC BY. Published by BMJ.

To cite: D'Souza N, Georgiou Delisle T, Chen M, et al. Gut 2021:70:1130-1138.

\begin{abstract}
Objective To assess whether a faecal immunochemical test (FIT) could be used to select patients with suspected colorectal cancer (CRC) symptoms for urgent investigation.

Design Multicentre, double-blinded diagnostic accuracy study in 50 National Health Service (NHS) hospitals across England between October 2017 and December 2019. Patients referred to secondary care with suspected CRC symptoms meeting NHS England criteria for urgent 2 weeks wait referral and triaged to investigation with colonoscopy were invited to perform a quantitative FIT. The sensitivity of FIT for CRC, and effect of relevant variables on its diagnostic accuracy was assessed. Results 9822 patients were included in the final analysis. The prevalence of CRC at colonoscopy was $3.3 \%$. The FIT positivity decreased from $37.2 \%$ to $19.0 \%$ and $7.6 \%$, respectively, at cut-offs of 2, 10 and $150 \mu \mathrm{g}$ haemoglobin $/ \mathrm{g}$ faeces $(\mu \mathrm{g} / \mathrm{g})$. The positive predictive values of FIT for CRC at these cut-offs were 8.7\% (95\% $\mathrm{Cl}, 7.8 \%$ to $9.7 \%), 16.1 \%(95 \% \mathrm{Cl} 14.4 \%$ to $17.8 \%)$ and $31.1 \%(95 \% \mathrm{Cl} 27.8 \%$ to $34.6 \%)$, respectively, and the negative predictive values were $99.8 \%(95 \% \mathrm{Cl}$ $99.7 \%$ to $99.9 \%)$, $99.6 \%(95 \% \mathrm{Cl} 99.5 \%$ to $99.7 \%$ ) and $98.9 \%$ (95\% CI $98.7 \%$ to $99.1 \%$ ), respectively. The sensitivity of FIT for CRC decreased at the same cut-offs from $97.0 \%(95 \% \mathrm{Cl} 94.5 \%$ to $98.5 \%)$ to $90.9 \%$ (95\% $\mathrm{Cl} 87.2 \%$ to $93.8 \%)$ and $70.8 \%(95 \% \mathrm{Cl} 65.6 \%$ to $75.7 \%$ ), respectively, while the specificity increased from $64.9 \%(95 \% \mathrm{Cl} 63.9 \%$ to $65.8 \%)$ to $83.5 \%$ (95\% Cl $82.8 \%$ to $84.3 \%$ ) and $94.6 \%$ (95\% Cl $94.1 \%$ to $95.0 \%)$, respectively. The area under the receiver operating characteristic curve was $0.93(95 \% \mathrm{Cl} 0.92$ to 0.95).
\end{abstract}

Conclusion FIT sensitivity is maximised to $97.0 \%$ at the lowest cut-off $(2 \mu \mathrm{g} / \mathrm{g})$; a negative FIT result at this cut-off can effectively rule out CRC and a positive FIT result is better than symptoms to select patients for urgent investigations.

Trial registration number ISRCTN49676259.

\section{INTRODUCTION}

Bowel symptoms are the imprecise basis of referral for urgent investigation in England to rule out cancer. ${ }^{12}$ Symptoms are non-specific for colorectal cancer (CRC); 96 of 100 patients referred urgently

\section{Significance of this study}

What is already known on this subject?

- Faecal immunochemical tests (FIT) are already recommended by the National Institute for Heath and Care Excellence to guide referral of patients with low-risk bowel symptoms but has not been recommended for all symptomatic patients due to concerns over the quality and power of previous studies.

What are the new findings?

- FIT sensitivity for colorectal cancer (CRC) is maximised to $97.0 \%$ at the limit of detection of $2 \mu \mathrm{g}$ haemoglobin $(\mathrm{Hb}) / \mathrm{g}$ faeces $(\mu \mathrm{g} / \mathrm{g})$.

- A faecal $\mathrm{Hb}$ concentration ( $f-\mathrm{Hb}$ ) result less than the limit of detection in symptomatic patients indicates that their chances of not having CRC is $99.8 \%$.

- There was no significant variation in the ability of FIT to detect CRC by patient or tumour characteristics, including age, sex, ethnicity, deprivation or iron-deficiency anaemia.

How might it impact on clinical practice in the foreseeable future?

- FIT could be used to rule out CRC in primary care for symptomatic patients meeting 2 weeks wait criteria, with sensitivity equivalent to colonoscopy at a cut-off of $2 \mu \mathrm{g} / \mathrm{g}$.

- FIT can be used to prioritise patients for investigation, as CRC and other serious bowel disease is more likely at higher $f-\mathrm{Hb}$ concentrations.

- The diagnostic accuracy of FIT for CRC is superior to symptoms.

on a 2 -week $(2 \mathrm{WW})$ wait pathway under National Institute for Health and Care Excellence (NICE) NG12 guidelines will not have CRC. ${ }^{1}$ Urgent referrals have increased by $90 \%$ over the last 5 years $^{3}$; $45 \%$ of UK endoscopy units are failing to meet CRC waiting targets. ${ }^{4}$

The faecal immunochemical test (FIT) was recommended by NICE (DG30) ${ }^{2}$ in 2017 to guide the referral of patients with low-risk symptoms of 
CRC, and is currently used in the National Health Service (NHS) of England. FIT detects the globin component of haemoglobin (Hb) by immunoassay and can reliably measure the faecal $\mathrm{Hb}$ concentration $(f-\mathrm{Hb})$ to the nearest microgram of $\mathrm{Hb}$ per gram of faeces $(\mu \mathrm{g} / \mathrm{g}) .^{5}$ Since 2010, over 25 diagnostic accuracy studies have reported data on the use of FIT in symptomatic patients utilising a range of cut-offs. ${ }^{6-8}$ In 2014, a study of 787 symptomatic patients from Spain suggested that FIT is more accurate for the detection of CRC than NICE 2005 criteria (CG27) although NICE have since expanded its referral criteria to include lower risk symptoms (NG12). ${ }^{9} 10$ More recently, two meta-analyses reported the sensitivity of FIT for CRC in symptomatic patients at a cut-off of $10 \mu \mathrm{g} / \mathrm{g}$ was $92.1 \%(95 \% \text { CI } 86.9 \% \text { to } 95.3 \%)^{6}$ and $94.1 \%$ (95\% CI 90.0\% to $96.6 \%){ }^{7}$ However, meta-analyses cannot account for variation in $\mathrm{f}$-Hb concentrations by patientlevel variables such as age, ${ }^{11-13}$ sex, ${ }^{11-13}$ deprivation ${ }^{13}{ }^{14}$ and between homogeneous ethnic population, ${ }^{15}$ which may lead to higher rates of undetected cancers within certain groups of patients. Consequently, a health technology assessment recommended that diagnostic cohort studies were performed to investigate variation in FIT accuracy in relevant subgroups. ${ }^{6}$ Similarly, a systematic review concluded a clear need for research on FIT as a triage test in the symptomatic primary care population. ${ }^{16}$

The NICE guidelines and FIT (NICE FIT) study was designed to investigate whether FIT could be used to rule out CRC in symptomatic patients in primary care meeting NICE $2 \mathrm{WW}$ criteria, and guide referral for further investigation.

\section{METHODS}

\section{Study design}

The study met Standards for Reporting of Diagnostic Accuracy Studies (STARD) guidelines. ${ }^{17}$ Ethics and study approval were granted from the UK Health Research Authority (IRAS 218404).
Patients were recruited at 50 NHS hospitals across England; sites were opened sequentially during the study.

The primary outcome measure was to identify a suitable $f-\mathrm{Hb}$ cut-off that would maximise sensitivity for CRC. The secondary outcome measures were to establish the diagnostic accuracy of FIT for CRC and other serious bowel disease (SBD) at different $f$-Hb cut-offs, and investigate the impact of other variables, such as age, sex, ethnicity and deprivation.

\section{Patient and public involvement}

Patient and public representatives were consulted through a process of in-depth interviews during the development of the study protocol. All relevant feedback was considered and incorporated into patient information sheets. Study progress and feedback was provided regularly to the Royal Marsden Partners (RM Partners) Patient Advisory Group by the senior research manager. The chief investigator regularly reported to the RM Partners Clinical Oversight Group which includedpatient and public involvement representatives throughout all phases of the study. The results will be disseminated to trial participants directly via email and the website (https://www.nicefitstudy. $\mathrm{com} /$ ), to other healthcare professionals at scientific conferences and through press releases.

\section{Patient selection}

All patients referred from primary care with symptoms of suspected CRC meeting NICE referral criteria under the 2WW pathway and who were triaged by secondary care clinicians to investigation by colonoscopy were eligible for inclusion. Secondary care sites were opened continuously throughout the process. The total number of eligible patients at each site was not captured but was dependent on the volume of referrals received by each site, and the length of time the study was

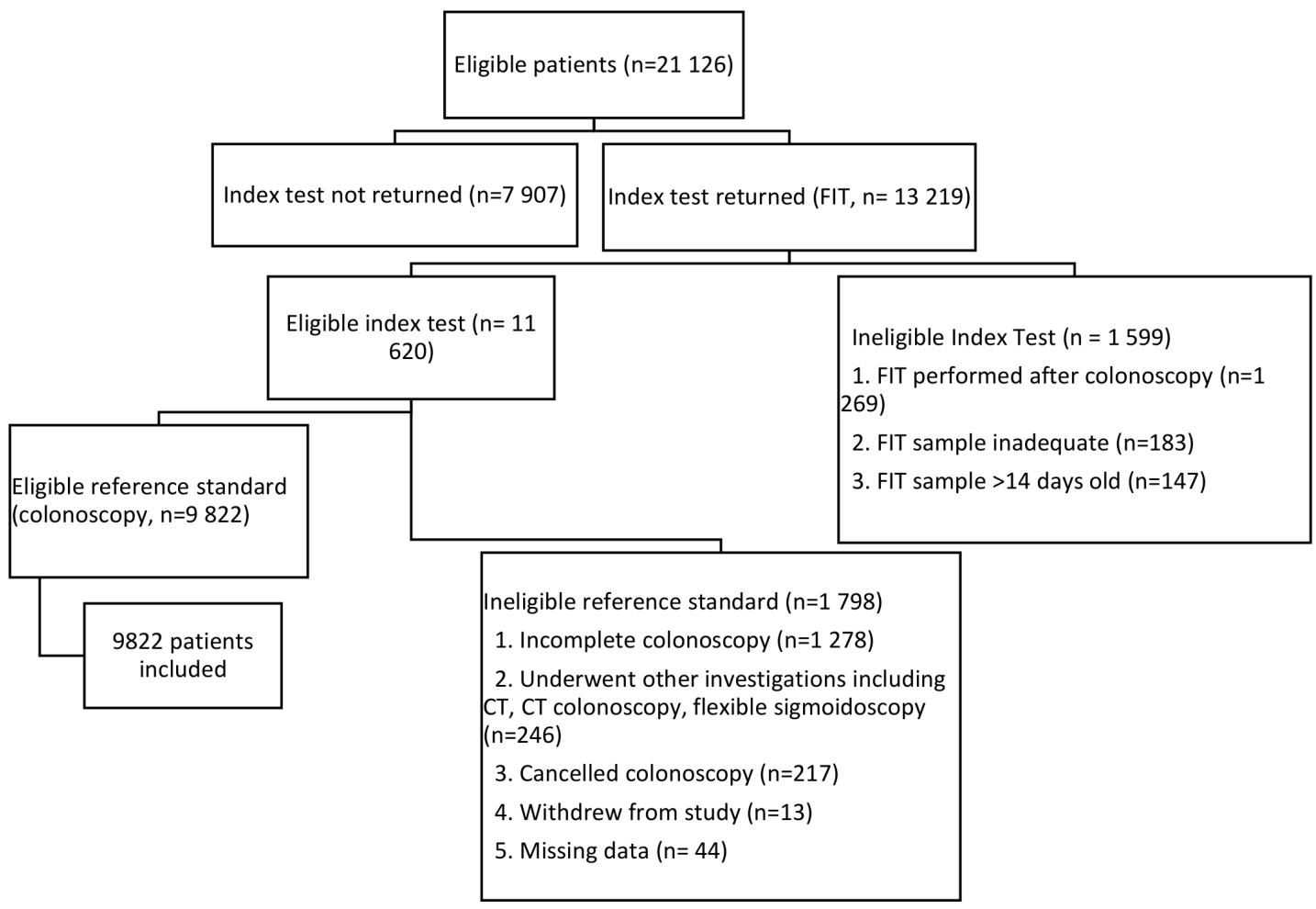

Figure 1 NICE FIT study flow diagram (adapted from STARD). FIT, faecal immunochemical test; NICE, National Institute for Heath and Care Excellence; STARD, Standards for Reporting of Diagnostic Accuracy Studies. 


\begin{tabular}{|c|c|c|}
\hline & $\mathrm{N}$ & $\%$ \\
\hline Total & 9822 & 100 \\
\hline \multicolumn{3}{|l|}{ Sex } \\
\hline Women & 5394 & 54.9 \\
\hline Men & 4428 & 45.1 \\
\hline \multicolumn{3}{|l|}{ Age (years) } \\
\hline Mean & 64.0 & \\
\hline SD & 11.9 & \\
\hline Minimum & 17 & \\
\hline Median & 65 & \\
\hline Maximum & 97 & \\
\hline \multicolumn{3}{|l|}{ Age group (years) } \\
\hline$<40$ & 361 & 3.7 \\
\hline $41-50$ & 940 & 9.6 \\
\hline $51-60$ & 2226 & 22.7 \\
\hline $61-70$ & 3033 & 30.9 \\
\hline$>70$ & 3262 & 33.2 \\
\hline \multicolumn{3}{|l|}{ Ethnicity } \\
\hline White & 7453 & 75.9 \\
\hline Asian & 614 & 6.3 \\
\hline Black & 365 & 3.7 \\
\hline Mixed & 58 & 0.6 \\
\hline Chinese & 42 & 0.4 \\
\hline Other* & 1103 & 11.2 \\
\hline \multicolumn{3}{|l|}{ Index of deprivation } \\
\hline Mean & 6.22 & \\
\hline SD & 2.62 & \\
\hline Median & 6 & \\
\hline \multicolumn{3}{|l|}{ Symptom risk category } \\
\hline High-medium (NG12) & 7194 & 73.2 \\
\hline Low (DG30) & 1994 & 20.3 \\
\hline Othert & 634 & 6.5 \\
\hline
\end{tabular}

*Other ethnicity: any other ethnic group, not-specified. tOther symptoms: patients referred urgently with symptoms of suspected CRC not meeting existing NG12 or DG30 criteria.

CRC, colorectal cancer.

open to recruitment. Data on symptoms were extracted from NICE NG12 2WW and DG30 referral criteria completed on the referral form by primary care clinicians. ${ }^{12}$ Patients referred urgently on a $2 \mathrm{WW}$ pathway without meeting NICE criteria

Table 2 Frequency of pathology findings at colonoscopy in symptomatic patients referred via $2 \mathrm{WW}$ pathways

\begin{tabular}{lll}
\hline Diagnosis & N & $\%$ \\
\hline Normal & 3079 & 31.3 \\
\hline Low risk adenoma & 2321 & 23.6 \\
Diverticular disease & 2294 & 23.4 \\
\hline Perianal disease* & 723 & 7.4 \\
Inflammatory bowel disease & 427 & 4.3 \\
\hline High-risk adenoma & 421 & 4.3 \\
Colorectal cancer & 329 & 3.3 \\
Microscopic colitis & 152 & 1.5 \\
Othert & 53 & 0.5 \\
Angiodysplasia & 23 & 0.2 \\
\hline
\end{tabular}

*Perianal disease: anal fissure, anal fistula, haemorrhoids or solitary rectal ulcer. tOther: findings included melanosis Coli, parasites, lipoma.

2WW, 2 weeks wait. due to clinical concerns were classified as 'others' and included in the analysis. Since patients are often referred with multiple symptoms or signs, a hierarchy was created to match one criterion to each patient, based on clinical estimation of positive predictive values (PPV). NG12 criteria were ranked in importance as follows: abdominal mass, iron-deficiency anaemia (IDA) (patients over 60 years), rectal bleeding, change in bowel habit (over 60) and abdominal pain and weight loss. DG30 criteria, were ranked in importance as follows: IDA (under 60), non-IDA, abdominal pain or weight loss, change in bowel habit (under 60).

Patients were identified by the central study team or local cancer research network (CRN) team once they had been booked for colonoscopy and contacted by post or telephone and invited to participate in the study. Patients were sent an FIT specimen collection device and asked to collect one sample of faeces prior to commencing bowel preparation for their colonoscopy. A first-class return envelope was enclosed for patients to post their sample directly to the study laboratory. Patients initially provided written consent, and after approval from the National Confidentiality Advisory Group, gave implied consent by returning an FIT sample.

Patients were not included if they did not return an FIT sample, did not have a complete colonoscopy unless due to CRC, were retriaged to another investigation (eg, flexible sigmoidoscopy or CT), or withdrew consent. Patients due to undergo colonoscopy within 3 days of identification were not invited to participate in the study, as there would not have been sufficient time to return a sample. In the original NG12 guidance, ${ }^{1}$ NICE recommended that patients with low risk bowel symptoms were tested with a guiac-based faecal occult blood test (gFOBT) prior to $2 \mathrm{WW}$ referral. In many regions, these patients were referred on $2 \mathrm{WW}$ pathways without gFOBT due to concerns over its poor sensitivity for $\mathrm{CRC},{ }^{18}$ and therefore, were eligible for inclusion. During this study, NICE recommended that low risk patients, as defined in DG30, ${ }^{2}$ were triaged in primary care with FIT prior to 2WW referral. This guidance was not fully implemented during this study, but those low-risk patients who were tested with FIT in primary care prior to referral were not included.

\section{Index test and reference standard}

FIT analysis was performed at one centralised laboratory where staff were blinded to patient clinical information. One HM-JACKarc analytical system (Hitachi Chemical Diagnostics Systems, Tokyo, Japan, supplied by Alpha Labs, Eastleigh, Hants, UK) was used to analyse all samples. The analytical working range is $7-400 \mu \mathrm{g} / \mathrm{g}$. The limit of detection (LoD) of the assay is $2 \mu \mathrm{g} / \mathrm{g}$ and the limit of quantitation is $7 \mu \mathrm{g} / \mathrm{g}$. NICE recommended an $f-H b$ cut-off of $10 \mu \mathrm{g} / \mathrm{g}$ in the DG30 guidelines. ${ }^{2}$ In accordance with previous publications on FIT, we chose the LoD and the $f$-Hb cut-off recommended in NICE DG30 as cut-offs to investigate sensitivity. To investigate the specificity and PPV at higher $f-\mathrm{Hb}$, we also chose a higher cut-off of $150 \mu \mathrm{g} / \mathrm{g}$ that had previously been reported to predict high rates of significant pathology. ${ }^{19}$ FIT specimen collection and handling, quality management and result handling was conducted and reported according to recent guidelines for studies on FIT $^{20}$ (see online supplemental appendix), using recommended analytical performance specifications. ${ }^{5}$ FIT samples that were unsuitable for analysis (collection device over or underfilled, or unavailable for analysis for more than 14 days) or performed after the colonoscopy were not included in the study.

Colonoscopy was chosen as the reference standard since it is acknowledged to be the gold-standard investigation for 
Table 3 Diagnostic accuracy of FIT for CRC at different cut-offs

\begin{tabular}{|c|c|c|c|c|c|c|c|c|c|c|}
\hline Cut-off $(\mu \mathrm{g} / \mathrm{g})$ & Positivity (\%) & NNS & Sensitivity (\%) & Specificity (\%) & PPV (\%) & NPV (\%) & TP & $\mathrm{FN}$ & FP & TN \\
\hline 2 & 37.2 & 11.5 & $97.0(94.5$ to 98.5$)$ & 64.9 (63.9 to 65.8$)$ & 8.7 (7.8 to 9.7) & 99.8 (99.7 to 99.9$)$ & 319 & 10 & 3336 & 6157 \\
\hline 10 & 19.0 & 6.2 & 90.9 (87.2 to 93.8$)$ & 83.5 (82.8 to 84.3 ) & 16.1 (14.4 to 17.8 ) & 99.6 (99.5 to 99.7$)$ & 299 & 30 & 1563 & 7930 \\
\hline 150 & 7.6 & 3.2 & 70.8 (65.6 to 75.7 ) & 94.6 (94.1 to 95.0$)$ & 31.1 (27.8 to 34.6 ) & 98.9 (98.7 to 99.1$)$ & 233 & 96 & 516 & 8977 \\
\hline$<2$ & 62.8 & 616.7 & 3 (1.5 to 5.5$)$ & 35.1 (34.2 to 36.1$)$ & $0.2(0.1$ to 0.3$)$ & 91.3 (90.3 to 92.2 ) & 10 & 319 & 6157 & 3336 \\
\hline
\end{tabular}

95\% Cls within brackets.

CRC, colorectal cancer; FIT, faecal immunochemical test; FN, false negatives; FP, false positives; NNS, number needed to scope; NPV, negative predictive value; PPV, positive predictive value; TN, true negatives; TP, true positives.

colorectal disease. Colonoscopists were blinded to the FIT results. Colonoscopy results were entered onto a secured online database designed specifically for the study by an external clinical research organisation (Hammersmith Medicines Research) and based on the national endoscopy logbook. Patients with incomplete colonoscopies (except when due to the presence of CRC) were excluded.

Clinical data extraction was performed initially by the local CRN team. A rigorous system of quality assurance was implemented. All colonoscopy and pathology results, as well as clinical and pathological tumour staging. were checked by the central study team, and then again by a team of senior colorectal clinicians blinded to the FIT laboratory results.

\section{Sample size}

To determine the sample size, calculations were based on a significance level of $5 \%$, power of $80 \%$ and prevalence of CRC within the NICE $2 \mathrm{WW}$ symptomatic population estimated at $3.5 \%$ based on data from the RM Partners Network. To demonstrate a lowest acceptable sensitivity of FIT for CRC of $98 \%$
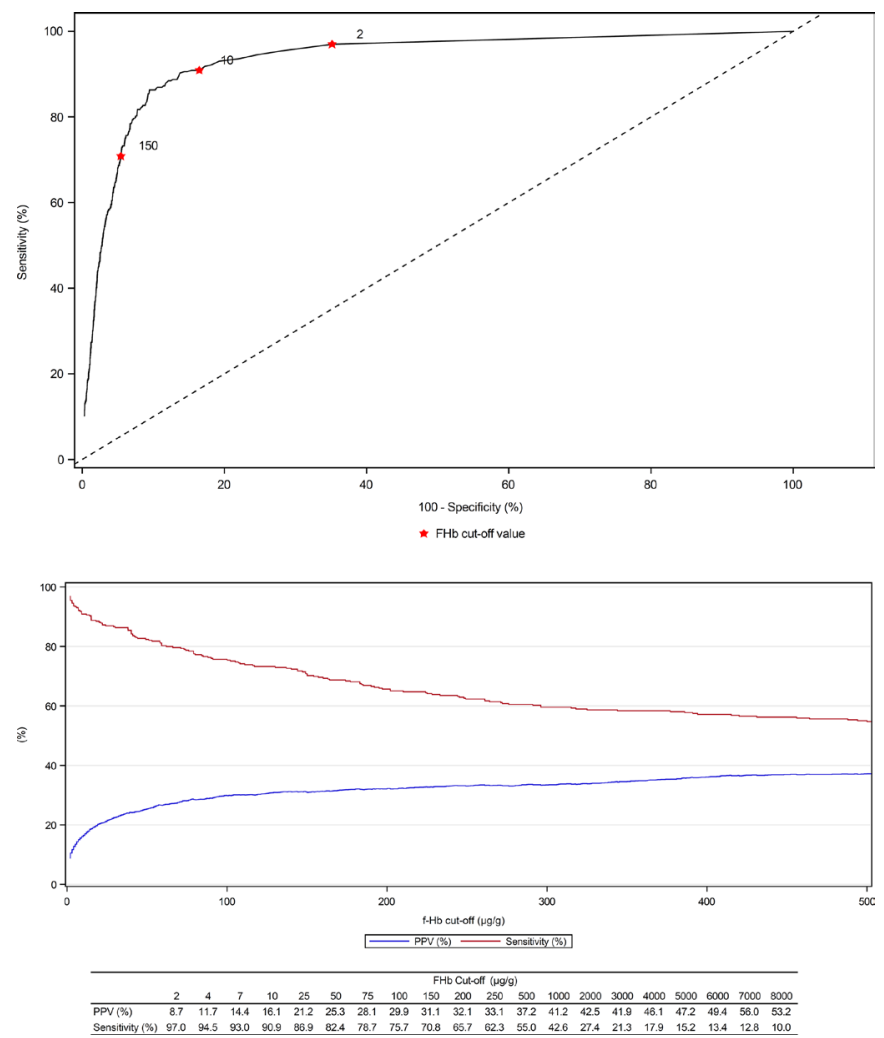

Figure 2 ROC curve (top) and PPV/sensitivity (bottom) of FIT for CRC. CRC, colorectal cancer; FIT, faecal immunochemical test; PPV, positive predictive value; ROC, receiver operating characteristic. with CI width of $2 \%$, a total sample size of 5379 patients was required. Given that previous studies had reported a 50\% noncompletion rate, it was determined that at least 10000 patients would need to be invited to participate in the study. The study was funded to over-recruit beyond this sample size to address the secondary endpoints and investigate the impact of other factors on FIT diagnostic accuracy. Accurate power calculations were not possible for the secondary endpoints, due to the lack of data on these covariates on the diagnostic accuracy of FIT for CRC in the symptomatic populations.

\section{Data analysis}

Patients with multiple findings at colonoscopy were recategorised with one diagnosis in a hierarchy; CRC ranked highest followed by high-risk adenoma (HRA) and then inflammatory bowel disease (IBD). These were grouped together as SBD. This was followed by low-risk adenoma (LRA) which was ranked above other non-malignant diagnoses, including diverticular disease, microscopic colitis, benign perianal disease (haemorrhoids, anal fissures, anal fistulas, solitary rectal ulcers), angiodysplasia, or rare findings such as melanosis coli, parasites or lipomas. HRA was defined by the NICE FIT Steering group as any polyp with high-grade dysplasia or polyps over $10 \mathrm{~mm}$ in size with low grade dysplasia, and serrated lesions in the right colon. Other polyps less than $10 \mathrm{~mm}$ were classified as LRA.

The indices of multiple deprivation were derived from postcodes $\left(1=\right.$ most deprived and $10=$ least deprived). ${ }^{21}$ Patients were classified as anaemic according to WHO criteria ${ }^{22}$; blood $\mathrm{Hb}$ concentration less than $120 \mathrm{~g} / \mathrm{L}$ for women or $130 \mathrm{~g} / \mathrm{L}$ for men, based on the most recent measurement within 3 months before referral. IDA was defined using British Society of Gastroenterology guidelines as present when serum ferritin concentration was less than $15 \mu \mathrm{g} / \mathrm{L}^{23}$

Data were assessed for normality by the Shapiro test and Q-Q plot analysis. Mann-Whitney and Kruskal Wallis tests were used for non-normally distributed data. Analysis of variancewas used across multiple groups, with separate models for each factor; age was pooled for analysis. Categorical data were compared with $\chi^{2}$ tests. Sensitivity, specificity, PPV and negative predictive value (NPV) were reported for each $f$-Hb cut-off, with $95 \%$ CIs . Receiver operating characteristic (ROC) curves were plotted for $f$-Hb. These were done using an initial threshold of 0.1 to calculate sensitivity and specificity, and then recalculated with increments of 0.1 to plot the ROC curve. In every statistical analysis, $\mathrm{p}<0.05$ was considered significant. All analyses were performed using SAS V.9.4 (SAS Institute).

\section{RESULTS}

Between October 2017 and December 2019, 21126 patients were sent recruitment packs, 13219 (62.6\%) returned FIT devices. Complete FIT and colonoscopy outcomes were available for 9822 patients, who were included in the study results. 
A study flow diagram is shown in figure 1: NICE FIT study flow diagram (adapted from STARD) . Data were not uploaded by the local sites for 44 patients were excluded.

Patient demographics are summarised in table 1 . The median patient age was 65.0 years (IQR 56.0-73.0). Women returned $54.9 \%$ of kits. The most common ethnic groups were white (75.9\%), other (11.2\%) and Asian (6.3\%). The median deprivation index score was 6.0 (IQR 4.0-9.0). Patients were referred most commonly with high-risk symptoms meeting NG12 criteria (73.2\%), followed by low-risk symptoms meeting DG30 criteria $(21.4 \%)$ or other symptoms warranting urgent referral $(6.4 \%)$.

Tests that were older than 14 days or sampled inadequately $(n=330)$ could not be analysed. FIT analysis was performed within 7 days of sample collection in $94.8 \%$ of specimens, and within a day of receipt by the laboratory in $94.6 \%$ of specimens.

Findings at colonoscopy are reported in table 2. Overall, the most prevalent finding at colonoscopy was that no disease was detected (31.3\%). SBD (CRC, HRA or IBD) was detected in $11.9 \%$ of patients during colonoscopy. CRC was detected in $3.3 \%$ of patients.

The diagnostic accuracy of FIT for CRC at $f-\mathrm{Hb}$ cut-offs of $2 \mu \mathrm{g} / \mathrm{g}, 10 \mu \mathrm{g} / \mathrm{g}$ and $150 \mu \mathrm{g} / \mathrm{g}$ are summarised in table 3 . The proportion of patients that had positive FIT results at $f$-Hb cutoffs of 2,10 and $150 \mu \mathrm{g} / \mathrm{g}$, respectively, decreased significantly $(\mathrm{p}<0.0001)$ from $37.2 \%$ to $19.0 \%$ and $7.6 \%$, respectively. At the same cut-offs, the PPV for CRC increased from 8.7\% (95\% CI, $7.8 \%$ to $9.7 \%$ ) to $16.1 \%$ (95\% CI $14.4 \%$ to $17.8 \%)$ and $31.1 \%$ (95\% CI $27.8 \%$ to $34.6 \%$ ), but the sensitivity for CRC declined from $97.0 \%$ (95\% CI $94.5 \%$ to $98.5 \%$ ) to $90.9 \%$ (95\% CI $87.2 \%$ to $93.8 \%$ ) and $70.8 \%$ (95\% CI $65.6 \%$ to $75.7 \%)$, as illustrated in figure 2 . The number needed to scope, ${ }^{24}$ that is, number of individuals required to undergo colonoscopy to detect 1 CRC was $11.5,6.2$ and 3.2 at $f$-Hb cut-offs of 2 , 10 and $150 \mu \mathrm{g} / \mathrm{g}$, compared with 29.9 for all patients referred on the current $2 \mathrm{WW}$ pathway. Some CRCs were not detected even a cut-off of $2 \mu \mathrm{g} / \mathrm{g}$, but significantly more CRCs (30 vs 10, $\mathrm{p}=0.0011)$ were not detected at a cut-off of $10 \mu \mathrm{g} / \mathrm{g}$. When $\mathrm{f}-\mathrm{Hb}$ was undetectable $(<2 \mu \mathrm{g} / \mathrm{g})$, the PPV for CRC was $0.2 \%(0.1 \%-$ $0.3 \%)$, and 617 patients would require colonoscopy to detect 1 CRC.

The diagnostic accuracy of FIT for SBD is summarised in table 4. The sensitivity of FIT for HRA and IBD are significantly lower than for CRC at every $f$-Hb cut-off. The PPV for SBD increases significantly at higher $f$-Hb cut-offs; $24.8 \%$ at $2 \mu \mathrm{g} / \mathrm{g}$, $39.6 \%$ at $10 \mu \mathrm{g} / \mathrm{g}$ and $64.5 \%$ at $150 \mu \mathrm{g} / \mathrm{g}$.

On ROC curve analysis (figure 2), the area under the curve (AUC) for CRC was 0.93 (0.92-0.95). Youden's index, which maximises the sum of sensitivity and specificity was $38 \mu \mathrm{g} / \mathrm{g}$, but FIT sensitivity was still optimised at $2 \mu \mathrm{g} / \mathrm{g}$.

Patients with CRC that had $f-\mathrm{Hb}<10 \mu \mathrm{g} / \mathrm{g}$ were analysed in further detail (table 5). There were no significant differences between patients with CRC and $f$-Hb greater or less than either cut-off of $2 \mu \mathrm{g} / \mathrm{g}$ or $10 \mu \mathrm{g} / \mathrm{g}$ with regard to age, sex, deprivation or ethnicity, iron and non-IDA or tumour characteristics.

\section{DISCUSSION}

This is the first powered, multicentre, double-blinded diagnostic accuracy study to demonstrate that FIT can be used to select patients with NICE $2 \mathrm{WW}$ symptoms for urgent investigation. FIT can be used to rule out CRC when $f-\mathrm{Hb}$ is undetectable or low. FIT sensitivity for CRC is significantly higher at $97 \%$ when using a lower $f$-Hb cut-off of the LoD $(2 \mu \mathrm{g} / \mathrm{g})$ compared with $10 \mu \mathrm{g} / \mathrm{g}$, the cut-off recommended in NICE DG30. No significant difference was found in FIT sensitivity on subgroup analysis by age, sex, deprivation, ethnicity and tumour characteristics, suggesting FIT can be used in all symptomatic patients that meet 2WW referral criteria. Employing a higher cut-off for investigation will result in a smaller group of FIT positive patients with a higher PPV or prevalence for CRC, but at the expense of detecting fewer CRC; this strategy may be adopted when endoscopy capacity is restricted or paused as occurred at the height of the current COVID-19 pandemic. ${ }^{25} 26$ The likelihood of cancer increases with increasing $f$-Hb concentrations (above $150 \mu \mathrm{g} / \mathrm{g}$ ), and consequently, FIT could be used to rule-in cancer or prioritise patients for investigation.

The most common finding at colonoscopy in symptomatic patients in our study was the absence of disease $(31.3 \%)$ in keeping with other reports on $2 \mathrm{WW}$ referrals ${ }^{19}{ }^{27}$; FIT can appropriately triage these patients off urgent pathways for investigation. Importantly, a negative FIT result can be used to reassure patients that their symptoms are unlikely to be due to CRC because of the high NPV; $99.8 \%$ and $99.6 \%$ at $2 \mu \mathrm{g} / \mathrm{g}$ and $10 \mu \mathrm{g} / \mathrm{g}$, respectively. Patients with symptoms meeting NICE criteria and negative FIT result at these cut-offs have less than $0.5 \%$ chance of CRC; a very low risk, but not no risk. In patients with undetectable $f-\mathrm{Hb}, 617$ patients would need to undergo

Table 4 Diagnostic accuracy of FIT for CRC and SBD at different cut-offs

\begin{tabular}{|c|c|c|c|c|c|c|}
\hline Risk category & FIT positivity & Cut-off $(\mu g / g)$ & Sensitivity & Specificity & PPV & NPV \\
\hline \multirow[t]{4}{*}{$\geq 2$} & 37.2 & CRC & 97.0 (94.5 to 98.5$)$ & 64.9 (63.9 to 65.8$)$ & 8.7 (7.8 to 9.7) & 99.8 (99.7 to 99.9 ) \\
\hline & & HRA & 65.8 (61.0 to 70.3$)$ & 64.1 (63.1 to 65.0$)$ & 7.6 (6.7 to 8.5$)$ & 97.7 (97.3 to 98.0 ) \\
\hline & & $\mathrm{IBD}$ & 73.1 (68.6 to 77.2$)$ & 64.4 (63.4 to 65.4$)$ & 8.5 (7.7 to 9.5$)$ & 98.1 (97.8 to 98.5 ) \\
\hline & & SBD & 77.1 (74.6 to 79.5$)$ & 68.2 (67.2 to 69.2$)$ & 24.8 (23.4 to 26.3 ) & 95.6 (95.1 to 96.1$)$ \\
\hline \multirow[t]{4}{*}{$\geq 10$} & 19.0 & CRC & 90.9 (87.2 to 93.8$)$ & 83.5 (82.8 to 84.3 ) & 16.1 (14.4 to 17.8$)$ & 99.6 (99.5 to 99.7 ) \\
\hline & & HRA & 45.4 (40.5 to 50.3$)$ & 82.2 (81.4 to 83.0$)$ & 10.3 (8.9 to 11.7$)$ & 97.1 (96.7 to 97.5 ) \\
\hline & & $\mathrm{IBD}$ & 57.8 (53.0 to 62.6$)$ & 82.8 (82.0 to 83.6 ) & $13.3(11.8$ to 14.9$)$ & 97.7 (97.4 to 98.1$)$ \\
\hline & & SBD & 62.6 (59.8 to 65.4$)$ & 87.0 (86.3 to 87.7 ) & 39.6 (37.4 to 41.8$)$ & 94.5 (93.9 to 95.0$)$ \\
\hline \multirow[t]{4}{*}{$\geq 150$} & 7.6 & CRC & 70.8 (65.6 to 75.7$)$ & 94.6 (94.1 to 95.0$)$ & 31.1 (27.8 to 34.6 ) & 98.9 (98.7 to 99.1$)$ \\
\hline & & HRA & 22.1 (18.2 to 26.4$)$ & 93.0 (92.5 to 93.5$)$ & 12.4 (10.1 to 15.0$)$ & 96.4 (96.0 to 96.8 ) \\
\hline & & $\mathrm{IBD}$ & 36.8 (32.2 to 41.5$)$ & 93.7 (93.2 to 94.2 ) & 21.0 (18.1 to 24.1$)$ & 97.0 (96.7 to 97.4 ) \\
\hline & & SBD & 41.0 (38.2 to 43.9$)$ & 96.9 (96.5 to 97.3 ) & 64.5 (60.9 to 67.9) & 92.4 (91.8 to 92.9 ) \\
\hline
\end{tabular}

95\% Cls within brackets.

CRC, colorectal cancer; FIT, faecal immunochemical test; HRA, high-risk adenoma; IBD, inflammatory bowel disease; NPV, negative predictive value; PPV, positive predictive value; $\mathrm{SBD}$, serious bowel disease. 
Table 5 Characteristics of 329 CRCs diagnosed in patients referred on a 2WW pathway overall, and classified by false negative FIT results at cutoffs of 2 or $10 \mu \mathrm{g} / \mathrm{g}$

\begin{tabular}{|c|c|c|c|c|c|c|c|c|}
\hline \multirow[b]{2}{*}{ Variable } & \multirow[b]{2}{*}{$\mathrm{n}$} & \multirow[b]{2}{*}{$\%$} & \multicolumn{2}{|c|}{$f$-Hb cut-off } & \multirow[b]{2}{*}{$P$ value } & \multicolumn{2}{|c|}{$f$-Hb cut-off } & \multirow[b]{2}{*}{$P$ value } \\
\hline & & & $\leq 2 \mu \mathrm{g} / \mathrm{g}$ & $>2 \mu \mathrm{g} / \mathrm{g}$ & & $\leq 10 \mu \mathrm{g} / \mathrm{g}$ & $>10 \mu \mathrm{g} / \mathrm{g}$ & \\
\hline \multicolumn{9}{|l|}{ Sex } \\
\hline Male & 199 & 60.5 & 6 & 193 & & 15 & 184 & \\
\hline \multicolumn{9}{|l|}{ Age group (years) } \\
\hline $30-49$ & 16 & 4.9 & 2 & 14 & 0.17 & 3 & 13 & 0.51 \\
\hline $70-79$ & 117 & 35.6 & 2 & 115 & & 13 & 104 & \\
\hline $80+$ & 44 & 13.4 & 1 & 43 & & 3 & 41 & \\
\hline \multicolumn{9}{|l|}{ Ethnicity } \\
\hline Asian & 15 & 4.6 & 0 & 15 & 0.87 & 2 & 13 & 0.87 \\
\hline Black & 8 & 2.4 & 0 & 8 & & 0 & 8 & \\
\hline Missing & 3 & 0.9 & 2 & 1 & & 2 & 1 & \\
\hline \multicolumn{9}{|l|}{ Deprivation } \\
\hline 1 & 6 & 1.8 & 0 & 6 & 0.51 & 1 & 5 & 0.13 \\
\hline 2 & 17 & 5.2 & 1 & 16 & & 1 & 16 & \\
\hline 3 & 26 & 7.9 & 0 & 25 & & 0 & 26 & \\
\hline 4 & 39 & 11.9 & 3 & 36 & & 7 & 32 & \\
\hline 5 & 36 & 10.9 & 2 & 34 & & 5 & 31 & \\
\hline 6 & 40 & 12.2 & 0 & 40 & & 2 & 38 & \\
\hline 7 & 41 & 12.5 & 0 & 41 & & 0 & 41 & \\
\hline 8 & 42 & 12.8 & 1 & 41 & & 5 & 37 & \\
\hline Adenocarcinoma & 269 & 86.9 & 9 & 277 & 0.28 & 28 & 286 & 0.74 \\
\hline Mucinous & 9 & 2.7 & 1 & 8 & & 1 & 8 & \\
\hline Other & 21 & 6.4 & 0 & 21 & & 1 & 20 & \\
\hline Missing & 13 & 4.0 & 0 & 13 & & 0 & 13 & \\
\hline \multicolumn{9}{|l|}{ Luminal narrowing } \\
\hline No & 103 & 31.3 & 3 & 100 & 0.73 & 12 & 91 & 0.26 \\
\hline Passable & 114 & 34.7 & 5 & 109 & & 12 & 102 & \\
\hline Impassable & 81 & 24.6 & 2 & 79 & & 4 & 77 & \\
\hline Missing & 81 & 24.6 & 0 & 31 & & 2 & 20 & \\
\hline \multicolumn{9}{|l|}{ Site } \\
\hline Caecum & 30 & 9.1 & 1 & 29 & 0.19 & 6 & 24 & 0.16 \\
\hline Ascending Colon & 45 & 13.7 & 1 & 44 & & 5 & 40 & \\
\hline Hepatic Flexure & 15 & 4.6 & 2 & 13 & & 3 & 12 & \\
\hline Transverse Colon & 14 & 4.3 & 1 & 13 & & 1 & 13 & \\
\hline Splenic Flexure & 5 & 1.5 & 1 & 4 & & 1 & 4 & \\
\hline Descending Colon & 8 & 2.4 & 0 & 8 & & 2 & 6 & \\
\hline Sigmoid Colon & 60 & 18.2 & 2 & 58 & & 4 & 56 & \\
\hline Rectosigmoid & 23 & 7.0 & 0 & 23 & & 1 & 22 & \\
\hline Rectum & 112 & 34.0 & 2 & 110 & & 6 & 106 & \\
\hline Anus & 5 & 1.5 & 0 & 5 & & 1 & 4 & \\
\hline Missing & 12 & 3.7 & 0 & 12 & & 0 & 12 & \\
\hline
\end{tabular}




\begin{tabular}{|c|c|c|c|c|c|c|c|c|}
\hline \multirow[b]{2}{*}{ Variable } & \multirow[b]{2}{*}{$\mathrm{n}$} & \multirow[b]{2}{*}{$\%$} & \multicolumn{2}{|c|}{$f$-Hb cut-off } & \multirow[b]{2}{*}{$P$ value } & \multicolumn{2}{|c|}{$f$-Hb cut-off } & \multirow[b]{2}{*}{$P$ value } \\
\hline & & & $\leq 2 \mu \mathrm{g} / \mathrm{g}$ & $>2 \mu \mathrm{g} / \mathrm{g}$ & & $\leq 10 \mu \mathrm{g} / \mathrm{g}$ & $>10 \mu \mathrm{g} / \mathrm{g}$ & \\
\hline 1 & 18 & 6.1 & 1 & 19 & 0.98 & 3 & 17 & 0.31 \\
\hline 2 & 64 & 19.5 & 2 & 62 & & 10 & 54 & \\
\hline 3 & 182 & 55.3 & 6 & 176 & & 14 & 168 & \\
\hline Unknown & 4 & 1.2 & 0 & 4 & & 0 & 4 & \\
\hline Missing & 18 & 5.5 & 0 & 18 & & 0 & 18 & \\
\hline \multicolumn{9}{|l|}{$\mathrm{N}$ stage } \\
\hline 0 & 158 & 53.2 & 4 & 154 & 0.71 & 16 & 142 & 0.44 \\
\hline 1 & 83 & 25.2 & 4 & 79 & & 11 & 72 & \\
\hline $1 c$ & 26 & 7.9 & 0 & 26 & & 1 & 25 & \\
\hline Yes & 128 & 38.9 & 2 & 126 & 0.65 & 7 & 121 & 0.13 \\
\hline No & 173 & 52.6 & 4 & 165 & & 18 & 155 & \\
\hline Missing & 28 & 8.5 & 4 & 28 & & 5 & 23 & \\
\hline \multicolumn{9}{|c|}{ Iron deficiency anaemia } \\
\hline Yes & 73 & 20.4 & 1 & 72 & 0.63 & 4 & 69 & 0.22 \\
\hline No & 173 & 51.4 & 4 & 169 & & 18 & 155 & \\
\hline Missing & 83 & 28.3 & 5 & 78 & & 8 & 75 & \\
\hline
\end{tabular}

Measures of association assessed by $\chi^{2}$.

CRC, colorectal cancer; FIT, faecal immunochemical test; 2WW, 2 weeks wait.

colonoscopy to detect $1 \mathrm{CRC}$; hence clinical acumen and safetynetting remains essential to identify patients with CRC and false negative FIT. ${ }^{28}$ The ROC AUC of 0.93 (0.92-0.95) confirms that the diagnostic accuracy of FIT is excellent, and on its own is at least as good as risk scores such as FAST (AUC 0.91) or COLONPREDICT (AUC 0.92) that combine FIT with other patient characteristics such as demographics, serum $\mathrm{Hb}$ and symptoms. ${ }^{29} 30$

Colonoscopy currently remains the gold-standard investigation to diagnose or exclude CRC but can fail to detect CRC. The sensitivity of colonoscopy for CRC in a meta-analysis of 9223 patients in 25 studies was $94.7 \%$ (95\% CI $90.4 \%$ to $92.7 \%$ ), although the largest trials reported data from asymptomatic participants in screening programmes. ${ }^{31} \mathrm{~A}$ recent study from the UK reported that the postcolonoscopy CRC rate at 3 years was $3.6 \%-7.4 \%$, implying that these CRC were potentially not detected at index colonoscopy. ${ }^{32}$ In this context, FIT sensitivity of $97.0 \%$ for CRC at a cut-off of the LoD appears to be equivalent to colonoscopy for the detection of CRC. Other SBDs such as HRA and IBD are associated with a raised $f-\mathrm{Hb}$; the PPV of $64.5 \%$ for SBD at $150 \mathrm{ug} / \mathrm{g}$ is clinically significant. However, the poor sensitivity of FIT for HRA at $65.8 \%$ and IBD at $73,1 \%$ at a cut-off of $2 \%$ and $45.4 \%$ and $57.8 \%$ at a cut-off of 10 suggest that FIT does not reliably identify these conditions.

FIT has already been recommended by NICE DG30 to triage patients with low-risk symptoms ${ }^{2}$ for investigation, but at the time was not been recommended for high-risk symptoms, due to a lack of robust evidence within the UK setting and because $f-\mathrm{Hb}$ are known to vary by age, ${ }^{11-13}$ sex, ${ }^{11-13}$ deprivation, ${ }^{13} 14$ cancer stage, ${ }^{33}$ IDA $^{1927}$ and between homogeneous ethnic populations. ${ }^{15}$ We investigated these known covariates and found that there was no significant difference in FIT sensitivity for CRC across all groups including cancer stage and IDA at cut-offs of 2 and $10 \mu \mathrm{g} / \mathrm{g}$. Previous studies have reported some differences in median $f$ - $\mathrm{Hb}$ across these variables ${ }^{11-15}$ but this was not clinically relevant for detection of CRC at the different cut-offs investigated. We have not found significant difference in FIT sensitivity in patients referred with IDA, as was reported in other studies. ${ }^{19}{ }^{27}$ However, missing data during referral from primary care or even prior to colonoscopy was common, particularly ferritin concentration (30\%), but even $\mathrm{Hb}$. Furthermore, data on luminal narrowing was not reported in $24.6 \%$ of colonoscopy reports, and while ethnic representation reflected the UK population, the numbers of some minority groups even within a study of this size remained small, and the potential for type II error exists.

Although not yet recommended by NICE, FIT is already being used by some services for high-risk symptoms. The largest two reports on the diagnostic accuracy of FIT in high risk symptoms were from service evaluations within Nottingham ${ }^{19}$ in England and NHS Tayside in Scotland. ${ }^{27}$ Neither study investigated the impact of age, sex, deprivation or ethnicity on FIT diagnostic accuracy. Chapman et al ${ }^{19}$ investigated 1106 patients in Nottingham with NICE NG12 2WW symptoms (excluding rectal bleeding) with FIT. Similar results to our study were reported, with sensitivity for CRC of $97.5 \%, 87.5 \%$ and $60 \%$ at cut-offs of $4 \mu \mathrm{g} / \mathrm{g}$ (the LoD of the FIT system used), $10 \mu \mathrm{g} / \mathrm{g}$ and $150 \mu \mathrm{g} / \mathrm{g}$, respectively; the PPV for CRC at the same cut-offs were $12.5 \%$, $14.6 \%$ and $35.8 \%$, respectively. No disease was found at colonoscopy in $58.8 \%$ of patients in Nottingham. In NHS Tayside, Mowat et $a l^{27}$ reported the results on 1447 symptomatic patients investigated with FIT prior to colonoscopy. At a cut-off of $10 \mu \mathrm{g} / \mathrm{g}$, the sensitivity of FIT was $90.5 \%$ and PPV was $11.0 \%$ : no disease was found in $27.8 \%$ of patients. FIT sensitivity for CRC in our study at $10 \mu \mathrm{g} / \mathrm{g}$ was similar to the results of previous meta-analyses of 4091 symptomatic patients 92.1\% (95\% CI $86.9 \%$ to $95.3 \%)^{6}$ and 6698 patients with specifically high-risk symptoms $91.7 \%$ (95\% CI $83.3 \%$ to $96.1 \%){ }^{7}$ However, given this prospective, multicentre research study is the largest to date, 
and designed to meet the highest methodological quality using STARD guidelines, our results on FIT accuracy unequivocally supports the use of FIT as a basis to triage patients with $2 \mathrm{WW}$ symptoms for referral and investigation.

Our system of quality assurance is the first described in the symptomatic FIT literature; over 30\% of errors in colonoscopy data coding were detected by clinicians, including misclassification of CRC. The missed CRC rate is unknown, since the volume and key performance indicators of individual endoscopists are unknown, although the majority of endoscopy units in this study were accredited by the Joint Advisory Group on Gastrointestinal Endoscopy. As 11\% of colonoscopies were incomplete and excluded from analysis, it is possible that the true prevalence of pathology present in a $2 \mathrm{WW}$ population was not captured by this study but at $3.3 \%$, CRC prevalence in this study is equivalent to CRC prevalence in the $2 \mathrm{WW}$ population recorded nationally. ${ }^{334}$

We found no obvious pattern or cause for false negative FIT results in patients with CRC, which may require further research into patient-level (genetic or medication) variables. Sampling studies in symptomatic patients (eg, multiple samples from the one bowel motion or consecutive motions) may provide possible strategies to improve sensitivity. Sequential use of further biomarkers (including volatile organic compounds in the urine, faeces or breath) following FIT might reduce the number of false positive and false negative results. ${ }^{35}{ }^{36}$ Our diagnostic accuracy results may not be replicated in other laboratories or FIT analysers, which may not be able to detect $f-\mathrm{Hb}$ down to $2 \mu \mathrm{g} / \mathrm{g}$; an international group is working on FIT method standardisation. ${ }^{37}$

Finally, the optimal FIT pathway remains unclear. When FIT was used in primary care in Scotland, referrals reduced by $15.1 \% .{ }^{27}$ When FIT accompanied referral in Nottingham, 2 WW referrals and 2WW CTC usage increased while there was no long-term reduction in $2 \mathrm{WW}$ colonoscopy usage; possibly due to referral of a wider, lower risk group of patients. ${ }^{38}$ We would recommend incorporating FIT into referral pathway of symptomatic patients in primary care with appropriate safety netting, to reduce unnecessary referrals for investigations and help secondary care prioritise patients with higher risk of CRC. NICE have already recommended in their DG30 guidance use of FIT in primary care as a triaging tool for low risk symptoms before referral to secondary care; this strategy should be expanded to include all symptomatic patients. The $f-\mathrm{Hb}$ cut-off for onwards referral should be set at the $\operatorname{LoD}(2 \mu \mathrm{g} / \mathrm{g})$ to provide sensitivity equivalent to colonoscopy, the current gold standard for investigation and yet reduce referrals by $60 \%$. While not the primary intention of the $2 \mathrm{WW}$ pathway, more cases of HRA (20.4\%) and IBD (15.3\%) will also be detected at the LOD than $10 \mu \mathrm{g} / \mathrm{g}$. Alternatively, the $f$-Hb cut-offs could be set higher to reduce referrals further to match existing colonoscopy resource and maximise the PPV for CRC.

\section{CONCLUSION}

FIT is superior to $2 \mathrm{WW}$ symptoms in predicting pathology in patients with suspected CRC. At a cut-off of the LoD of the FIT analytical system used, FIT detects CRC with equivalent diagnostic accuracy to colonoscopy. A higher $f$-Hb cut-off may be set to match capacity in resource-limited settings; this will reduce the number of positive results, onwards referral for investigation and demand for colonoscopy but at the expense of detecting fewer cancers. High $f$-Hb levels are associated with a high PPV for CRC and SBD and can be used to prioritise investigations.

Correction notice This article has been corrected since it published Online First. The formatting of the last column in table 5 has been corrected.
Twitter Nigel D'Souza @mrnigeldsouza and Muti Abulafi @muti192

Acknowledgements Patients were recruited nationally through the NIHR Clinical Research Network, Principal Investigators and R\&D teams at each site. The Southern Bowel Cancer Screening Hub Research Team provided analytical expertise and advice, in addition to analysing all samples. Hammersmith Medical Research provided expert database management and statistical analysis. Callum $G$ Fraser expertly revised drafts of the manuscript. Alpha Labs supported the study prior to funding with FIT kits and production of patient information. The NICE FIT steering committee comprised: Oliver Warren (Chelsea and Westminster Hospital NHS Foundation Trust); Saidyousuf Ahmadi, Carlene Parchment, Arun Shanmuganandan (Croydon University Hospital); Nicholas West (Epsom and St Helier University Hospitals); Toni Mitchell, Stephen Sah and Nick Jackson (Hammersmith Medical Research); Alistair Myers (Hillingdon Hospitals NHS Foundation Trust); Paul Ziprin (Imperial College Healthcare NHS Trust); Ian Bloom (Kingston Hospital NHS Foundation Trust); Stan Kaye (Royal Marsden Partners); Andy Ramwell (St George's University Hospitals NHS Foundation Trust); Kevin Monahan (West Middlesex University Hospital). Principal Investigators: Pasha Nisar (Ashford and St Peter's Hospitals NHS Foundation Trust); Yuksel Gercek (Bedford Hospital NHS Trust); Mark Austin (Brighton and Sussex University Hospitals NHS Trust); Nigel Hall (Cambridge University Hospitals NHS Foundation Trust); Robin Gupta (Chesterfield Royal Hospital NHS Foundation Trust); Rakesh Bhardway (Dartford and Gravesham NHS Trust); Arabis Oglesby (Dorset County Hospital NHS Foundation Trust); Usman Khan (East Cheshire NHS Trust); Nasser Khan (East and North Hertfordshire NHS Trust); Sudhakar Mangam (East Kent Hospitals University NHS Foundation Trust); Alexandra Cope (Frimley Health NHS Foundation Trust); Isobel Thomas (United Lincolnshire Hospitals NHS Trust); John Ramage (Hampshire Hospitals NHS Foundation Trust); Mark Pugh (Isle of Wight NHS Trust); Roshan Lal (James Paget University Hospitals NHS Foundation Trust); Tushar Agarwal, Ian Jenkins (London North West University Healthcare); Mark Hill (Maidstone and Tunbridge Wells NHS Trust); Henk Wegstapel (Medway NHS Foundation Trust); Vimal Hariharan (North West Anglia NHS Foundation Trust); Salim Kurrimboccus (Pennine Acute Hospitals NHS Trust); Andrew Clarke (Poole Hospital NHS Foundation Trust); Tracey Dobson (Portsmouth Hospitals NHS Trust); Joseph Ellul (Princess Royal University Hospital, King's College Hospital NHS Foundation Trust); Hayley Webb (Queen Elizabeth Hospital Kings Lynn NHS Trust); Gemma Faulkner (Bolton NHS Foundation Trust); Sanjaya Wijeyekoon (Royal Bournemouth and Christchurch Hospitals NHS Foundation Trust); Neil Smart (Royal Devon \& Exeter NHS Foundation Trust); John Stebbing (Royal Surrey NHS Foundation Trust); Jonathan Epstein (Salford Royal NHS Foundation Trust); Graham Branagan (Salisbury NHS Foundation Trust); Stephen Foley (Sherwood Forest Hospitals NHS Foundation Trust); Andrew Bateman (University Hospital Southampton NHS Foundation Trust); Bruce Levy (Western Sussex Hospitals Foundation Trust); Andrew Day (Surrey and Sussex Healthcare NHS Trust); Fionnuala Lenehan (Walsall Healthcare NHS Trust); Nicholas Ward (West Suffolk NHS Foundation Trust); Lisa Wacklin (Weston Area Health NHS Trust); Naomi Mackenzie (Wrightington, Wigan and Leigh NHS Foundation Trust).

Collaborators The NICE FIT Steering Group: Oliver Warren; Saidyousuf Ahmadi; Carlene Parchment; Arun Shanmuganandan; Nicholas West; Toni Mitchell; Stephen Sah; Nick Jackson; Alistair Myers; Paul Ziprin; Ian Bloom; Stan Kaye; Andy Ramwell; John T Jenkins; Kevin Monahan.

Contributors ND and MA were responsible for conception and design of the study. ND, TGD, MC, SB and MA were responsible for data analysis and interpretation, and critical revision of the manuscript. The trial steering group provided oversight of recruitment activity, data analysis and interpretation. All authors were responsible for data acquisition. MA is the guarantor of the study, and affirms that the manuscript is an honest, accurate and transparent account of the study being reported; that no important aspects of the study have been omitted; and that any discrepancies from the study as originally planned (and, if relevant, registered) have been explained.

Funding This study was funded by NHS England awarded to RM Partners, the West London Cancer Alliance hosted by The Royal Marsden NHS Foundation Trust. The study is supported by the National Institute for Health Research Clinical Research Network Portfolio. Croydon University Hospital acted as study sponsor. Alpha Labs Ltd, particularly Matthew Davis and Emma Boxall, supported the study by providing FIT collection devices without charge. All FIT kits and reagents were provided by Alpha Labs Ltd. The study was supported by Croydon University Hospital and RM Partners in the design and conduct of the trial.

Disclaimer The study sponsors and funders had no role in study design; in the collection, analysis, and interpretation of data; in the writing of the report; and in the decision to submit the article for publication.

Competing interests All authors have completed the ICMJE uniform disclosure form at www.icmje.org/coi_disclosure.pdf.

Patient and public involvement Patients and/or the public were involved in the design, or conduct, or reporting, or dissemination plans of this research. Refer to the Methods section for further details.

Patient consent for publication Not required 
Ethics approval The study was approved by the National Research Ethics Service Committee, London- South East (reference 16/LO/2174). Ethics and study approval were granted from the UK Health Research Authority (IRAS 218404).

Provenance and peer review Not commissioned; externally peer reviewed. Data availability statement No data are available.

Open access This is an open access article distributed in accordance with the Creative Commons Attribution 4.0 Unported (CC BY 4.0) license, which permits others to copy, redistribute, remix, transform and build upon this work for any purpose, provided the original work is properly cited, a link to the licence is given, and indication of whether changes were made. See: https://creativecommons.org/ licenses/by/4.0/.

\section{ORCID iDs}

Nigel D'Souza http://orcid.org/0000-0002-9614-6113

Muti Abulafi http://orcid.org/0000-0001-7088-4292

\section{REFERENCES}

1 NICE. Suspected cancer: recognition and referral [NG 12]. National Institute for Health and Care Excellence, 2015.

2 NICE. Diagnostics quidance [DG30]. Quantitative faecal immunochemical tests to guide referral for colorectal cancer in primary care. National Institute for Health and Care Excellence, 2017.

3 NHS England. Cancer waiting times annual reports. NHS England and NHS Improvement, 2019.

4 Shenbagaraj L, Thomas-Gibson S, Stebbing J, et al. Endoscopy in 2017: a national survey of practice in the UK. Frontline Gastroenterol 2019;10:7-15.

5 Fraser CG, Benton SC. Detection capability of quantitative faecal immunochemical tests for haemoglobin (FIT) and reporting of low faecal haemoglobin concentrations. Clin Chem Lab Med 2019:57:611-6.

6 Westwood M, Corro Ramos I, Lang S, et al. Faecal immunochemical tests to triage patients with lower abdominal symptoms for suspected colorectal cancer referrals in primary care: a systematic review and cost-effectiveness analysis. Health Technol Assess 2017:21:1-234.

7 Pin Vieito N, Zarraquiños S, Cubiella J. High-risk symptoms and quantitative faecal immunochemical test accuracy: systematic review and meta-analysis. World J Gastroenterol 2019;25:2383-401.

8 Mowat C, Digby J, Strachan JA, et al. Faecal haemoglobin and faecal calprotectin as indicators of bowel disease in patients presenting to primary care with bowel symptoms. Gut 2016;65:1463-9.

9 NICE. Referral quidelines for suspected cancer [CG27]. National Institute for Clinical Excellence, 2005

10 Cubiella J, Salve M, Díaz-Ondina M, et al. Diagnostic accuracy of the faecal immunochemical test for colorectal cancer in symptomatic patients: comparison with NICE and SIGN referral criteria. Colorectal Dis 2014;16:0273-82.

11 McDonald PJ, Strachan JA, Digby J, et al. Faecal haemoglobin concentrations by gender and age: implications for population-based screening for colorectal cancer. Clin Chem Lab Med 2012;50:935-40.

12 Arana-Arri E, Idigoras I, Uranga B, et al. Population-based colorectal cancer screening programmes using a faecal immunochemical test: should faecal haemoglobin cut-offs differ by age and sex? BMC Cancer 2017;17:577.

13 Symonds EL, Osborne JM, Cole SR, et al. Factors affecting faecal immunochemical test positive rates: demographic, pathological, behavioural and environmental variables. J Med Screen 2015:22:187-93.

14 Digby J, McDonald PJ, Strachan JA, et al. Deprivation and faecal haemoglobin: implications for bowel cancer screening. J Med Screen 2014;21:95-7.

15 Fraser CG, Rubeca T, Rapi S, et al. Faecal haemoglobin concentrations vary with sex and age, but data are not transferable across geography for colorectal cancer screening. Clin Chem Lab Med 2014:52:1211-6.

16 van Melle M, Yep Manzano SI, Wilson $\mathrm{H}$, et al. Faecal immunochemical test to triage patients with abdominal symptoms for suspected colorectal cancer in primary care: review of international use and guidelines. Fam Pract 2020. doi:10.1093/fampra/ cmaa043. [Epub ahead of print: 07 May 2020].

17 Bossuyt PM, Reitsma JB, Bruns DE, et al. STARD 2015: an updated list of essential items for reporting diagnostic accuracy studies. Radiology 2015;277:826-32.

18 Steele R, Forgacs I, McCreanor G, et al. Use of faecal occult blood tests in symptomatic patients. BMJ 2015;351:h4256.

19 Chapman C, Bunce J, Oliver S, et al. Service evaluation of faecal immunochemical testing and anaemia for risk stratification in the 2-week-wait pathway for colorectal cancer. BJS Open 2019;3:395-402.

20 Fraser CG, Allison JE, Young GP, et al. A standard for faecal immunochemical tests for haemoglobin evaluation reporting (FITTER). Ann Clin Biochem 2014;51:301-2.

21 Smith T, Noble M, Noble S, et al. The English indices of deprivation 2015. London: Department for Communities and Local Government, 2015.

22 World Health Organisation. Haemoglobin concentrations for the diagnosis of anaemia and assessment of severity. Geneva: World Health Organisation, 2011.

23 Goddard AF, James MW, McIntyre AS, et al. Guidelines for the management of iron deficiency anaemia. Gut 2011;60:1309-16.

24 van Rossum LGM, van Rijn AF, Laheij RJF, et al. Cutoff value determines the performance of a semi-quantitative immunochemical faecal occult blood test in a colorectal cancer screening programme. Br I Cancer 2009;101:1274-81.

25 Yeo C, Kaushal S, Yeo D. Enteric involvement of coronaviruses: is faecal-oral transmission of SARS-CoV-2 possible? Lancet Gastroenterol Hepatol 2020;5:335-7.

26 BSG. Endoscopy activity and COVID-19: BSG and JAG guidance, 2020. Available: https://www.bsg.org.uk/covid-19-advice/endoscopy-activity-and-covid-19-bsg-andjag-guidance/

27 Mowat C, Digby J, Strachan JA, et al. Impact of introducing a faecal immunochemical test (FIT) for haemoglobin into primary care on the outcome of patients with new bowel symptoms: a prospective cohort study. BMJ Open Gastroenterol 2019;6:e00293.

28 Cancer Research UK. Early diagnosis of cancer how do we make sure patients don't slip through the net? Oxford: Cancer Research UK, 2016

29 Cubiella J, Vega P, Salve M, et al. Development and external validation of a faecal immunochemical test-based prediction model for colorectal cancer detection in symptomatic patients. BMC Med 2016;14:128

30 Cubiella J, Digby J, Rodríguez-Alonso L, et al. The fecal hemoglobin concentration, age and sex test score: development and external validation of a simple prediction tool for colorectal cancer detection in symptomatic patients. Int I Cancer 2017;140:2201-11.

31 Pickhardt PJ, Hassan C, Halligan S, et al. Colorectal cancer: CT colonography and colonoscopy for detection--systematic review and meta-analysis. Radiology 2011:259:393-405.

32 Burr NE, Derbyshire E, Taylor J, et al. Variation in post-colonoscopy colorectal cancer across colonoscopy providers in English National health service: population based cohort study. BMJ 2019;367:16090.

33 Niedermaier T, Weigl K, Hoffmeister $\mathrm{M}$, et al. Fecal immunochemical tests in combination with blood tests for colorectal cancer and advanced adenoma detectionsystematic review. United European Gastroenterol I 2018;6:13-21.

34 NCRAS. Routes to diagnosis 2006-2016 year breakdown. Public Health England, 2018

35 Widlak MM, Neal M, Daulton E, et al. Risk stratification of symptomatic patients suspected of colorectal cancer using faecal and urinary markers. Colorectal Dis 2018;20:0335-42.

36 Fraser CG. Faecal immunochemical tests for haemoglobin (FIT) in the assessment of patients with lower abdominal symptoms: current controversies. Gastroenterol Hepatol 2019:42:263-70.

37 The International Federation of Clinical Chemistry and Laboratory Medicine. Fecal Immunochemical Testing (WG-FIT) [Working Group], 2020. Available: https://www. ifcc.org/ifcc-scientific-division/sd-working-groups/wg-fit/

38 Bailey JA, Khawaja A, Andrews H, et al. GP access to FIT increases the proportion of colorectal cancers detected on urgent pathways in symptomatic patients in Nottingham. Surgeon 2020. doi:10.1016/j.surge.2020.03.002. [Epub ahead of print: 20 Apr 2020]. 